\title{
Preparation of New Binaphthol Derived Chiral Stationary Phases
}

\author{
Yi Jun Zhang ${ }^{\dagger, *}$ and Jae Jeong Ryoo ${ }^{\dagger, \S, *}$ \\ ${ }^{\dagger}$ Department of Chemistry, Graduate School, Kyungpook National University, Daegu 702-701, Korea \\ Henan Institute of Science and Technology, Xinxiang 453003, P.R. China \\ ${ }^{\S}$ Department of Chemistry Education and Green-Nano Materials Research Center, \\ Kyungpook National University, Daegu 702-701, Korea.*E-mail: jjryoo@knu.ac.kr \\ Received May 12, 2011, Accepted July 6, 2011
}

\begin{abstract}
To develop more advanced binaphthol derived CSPs, two new 1,1-bi-2-naphthol derived chiral stationary phases (CSPs) (CSP 1 and CSP 2) were prepared by connecting with silica gel at a hydroxy (OH) group of binaphthol. Previously reported two 1,1'-binaphthyl-2,2'-diamine derived CSPs (CSP 3 and CSP 4) were also prepared to compare the role of their functional groups $\left(-\mathrm{OH}\right.$ and $\left.-\mathrm{NH}_{2}\right)$ with $\mathbf{C S P} \mathbf{1}$ and CSP 2. Enantioseparation of randomly selected, 11 chiral compounds on these four CSPs was performed and the results compared to each other. 3,5-Dinitrobenzoyl (3,5-DNB) derivatives of each CSP showed better results than the others and binaphthyldiamine derived CSP showed slightly better than binaphthol derived one.
\end{abstract}

Key Words : Binaphthol chiral stationary phase, Enantioseparation

\section{Introduction}

The increased demand for optically pure chiral compounds has led to the development of various asymmetric synthesis methods ${ }^{1,2}$ and a variety of chiral separation technologies. ${ }^{1,3}$ Among the methods of obtaining optically pure or active chiral compounds, direct chiral liquid chromatographic method by using various chiral stationary phases is the most important, because it can provide optically pure compounds directly and the information of optical purities of the optically active chiral compounds obtained from an asymmetric synthesis reaction. ${ }^{3}$ Various chiral stationary phases have been developed to separate as many chiral compounds as possible with high selectivity. ${ }^{3}$ Many small chiral molecules were used as a candidate of a good chiral selector in the course of the development of a powerful chiral stationary phase. ${ }^{1,3}$

Recently, optically pure 1,1'-binaphthyl-2,2'-diamine was used as a chiral selector, and four binaphthyldiamine derived chiral stationary phases (CSPs) were prepared and used for chiral separation of some chiral compounds. ${ }^{4,5} 1,1$ '-Bi-2naphthol also used a powerful chiral catalyst ${ }^{6,7}$ or a chiral derivatizing agent ${ }^{8}$ for asymmetric synthesis. Sudo et al. reported four binaphthol derived CSPs (CSP 5-8) by connecting with silica gel at 6-position of binaphthol and showed very poor enantioselectivities for 23 chiral samples. ${ }^{9}$

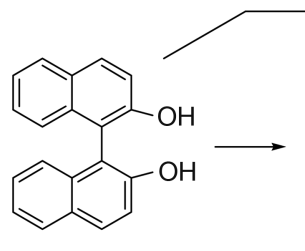

1,1'-bi-2-naphthol<smiles>[R15]Oc1ccc2ccccc2c1-c1c(OC(=O)NCCCC2(CC)O[Si](CC)(OCC)O2)ccc2ccccc12</smiles>

CSP1

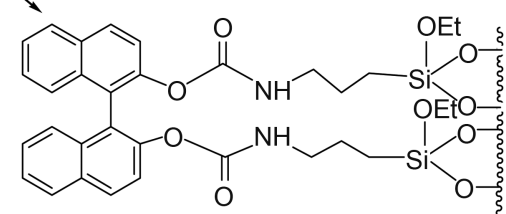

CSP2

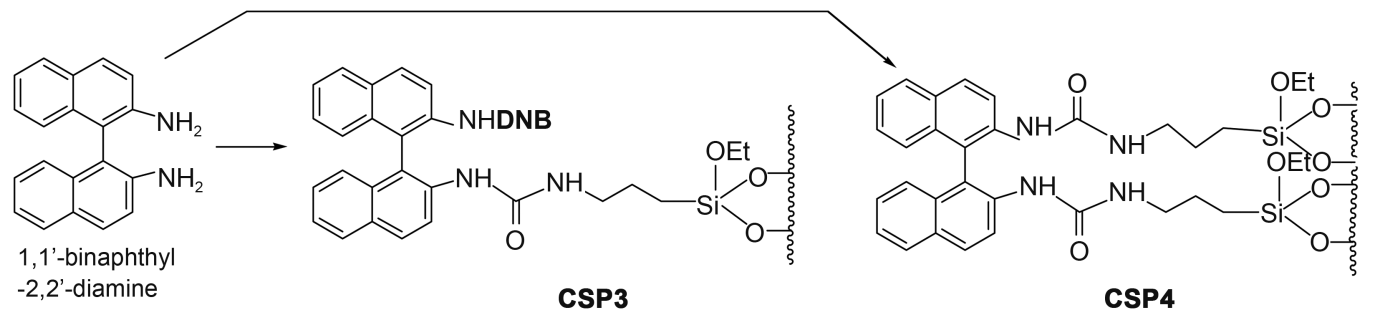<smiles></smiles> 
In this study, two 1,1'-bi-2-naphthol derived CSPs (CSP 1 and CSP 2) were newly prepared by connecting with silica gel at a hydroxy $(\mathrm{OH})$ group of binaphthol to compare the chiral separation results from CSP 5-8 and application of the CSP 1 and CSP 2 for the separation of some randomly selected eleven chiral compounds will be shown. For an exact comparison between these CSPs and the previously reported two 1,1'-binaphthyl-2,2-diamine derived CSPs (CSP 3 and CSP 4), we prepared the CSP 3 and CSP 4 again under similar synthetic procedure of CSP 1 and CSP 2 and compared the chiral separation results of the eleven chiral compounds on these columns.

\section{Experimental}

${ }^{1} \mathrm{H}-\mathrm{NMR}$ spectra were recorded on a Bruker AVANCE digital 400 Spectrometer (400 MHz). IR spectra were measured with a Thermo Nicolet IR 200 FT-IR Spectrometer. Elemental analysis data were obtained using a ThermoFisher Flash 2000 Elemental Analyzer. All reactions were performed under a nitrogen atmosphere.

The HPLC system consisted of a JASCO (Tokyo, Japan) PU-2080 Plus Intelligent HPLC pump, a Rheodyne (Cotati, CA, USA) Model $7725 \mathrm{i}$ injector with a $20 \mu \mathrm{L}$ sample loop, a UV-2075 Plus Intelligent UV-Vis Detector. All chromatographic data were obtained using 2.5-10\% 2-propanol (IPA) in hexane, as a mobile phase at a flow rate of $0.3-1.0 \mathrm{~mL} /$ min. Dead time $\left(\mathrm{t}_{0}\right)$ was checked by injecting 1,3,5-tri-tertbutylbenzene, a presumed unretained solute obtained from Aldrich Chemical Co. Chiral Columns: CSP 1 was packed into $2.1 \mathrm{~mm}$ I.D. $\times 150 \mathrm{~mm}$ stainless steel column, while CSP 2, CSP 3 and CSP 4 were packed into $4.6 \mathrm{~mm}$ I.D. $\times 150 \mathrm{~mm}$ stainless steel column. (S)-1,1'-Binaphthyl-2,2'diamine, $(R)$-1,1'-bi-2-naphthol, 3-(triethoxysilyl)propyl isocyanate, 3,5-dinitrobenzoyl chloride and an underivatized racemic sample (S1) were purchased from Sigma-Aldrich Korea (Seoul, Korea). Spherical silica gel $(5 \mu \mathrm{m})$ was purchased from Merck Korea (Seoul, Korea). Other racemic samples (S2-S11) were obtained from Prof. DW Armstrong's lab. (U. Texas, Arlington, USA) or Prof. MH Hyun's lab. (Pusan Nat'l Univ., Korea) The structures of chiral samples used in this study are shown in Figure 1.

\section{Preparation of CSP 1.}

Preparation of 2'-hydroxy-[1,1'-binaphthalen]-2-yl 3,5dinitrobenzoate (1): $(R)-1,1^{\prime}-\mathrm{Bi}(2-$ naphthol $)(0.60 \mathrm{~g}, 2.07$ $\mathrm{mmol})$ and triethylamine $(0.28 \mathrm{~mL}, 2.07 \mathrm{mmol})$ were dissolved in $100 \mathrm{~mL}$ of dry methylene chloride in a $250 \mathrm{~mL}$ two-necked round bottom flask. To the stirred solution, a solution of 3,5-dinitrobenzoyl chloride (DNB-Cl) $(0.49 \mathrm{~g}$, $1.73 \mathrm{mmol}$ ) in $50 \mathrm{~mL}$ dry methylene chloride was dropped as slowly as possible under nitrogen at $0{ }^{\circ} \mathrm{C}$. The reaction mixture was stirred at room temperature under nitrogen for $6 \mathrm{~h}$ and then washed with $1.0 \mathrm{M} \mathrm{HCl}$, saturated aqueous $\mathrm{NaHCO}_{3}$, and brine. The organic solution was dried over anhydrous $\mathrm{MgSO}_{4}$, filtered and evaporated. The residue was purified by flush column chromatography over silica gel with EtOAc/n-hexane (1:6) to afford $1(0.90 \mathrm{~g}$, yield $90 \%)$ as a yellow powder. ${ }^{1} \mathrm{H}-\mathrm{NMR}\left(\mathrm{CDCl}_{3}\right) \delta$ 7.0-7.7(m, $\left.12 \mathrm{H}\right)$, 8.41-8.43 (d, 1H), $8.6(\mathrm{~d}, 2 \mathrm{H}), 9.1(\mathrm{t}, 1 \mathrm{H})$. IR $(\mathrm{KBr}) \mathrm{cm}^{-1}$ : 3484, 3079, 2925, 2855, 1748, 1543, 1344.

Preparation of 2'-(((3-(triethoxysilyl)propyl)carbamoyl)oxy)-[1,1'-binaphthalen]-2-yl 3,5-dinitrobenzoate (2): 3-(Triethoxysilyl)-propyl isocyanate $(0.46 \mathrm{~mL}, 1.78 \mathrm{mmol})$ and triethylamine $(0.25 \mathrm{~mL}, 1.78 \mathrm{mmol})$ were added to a stirred solution of $1(0.85 \mathrm{~g}, 1.78 \mathrm{mmol})$ in $100 \mathrm{~mL}$ dry methylene chloride. The whole reaction mixture was stirred for $72 \mathrm{~h}$ under reflux, and then the solvent was evaporated. The residue was purified by column chromatography over silica gel with EtOAc/n-hexane/ $\mathrm{CH}_{2} \mathrm{Cl}_{2}(1: 4: 10)$ to afford 2 $(0.65 \mathrm{~g}$, yield $50.3 \%)$ as a yellow powder. ${ }^{1} \mathrm{H}-\mathrm{NMR}\left(\mathrm{CDCl}_{3}\right)$<smiles>OC(c1c2ccccc2cc2ccccc12)C(F)(F)F</smiles>

S1<smiles>CCN(CC)C(=O)C(CC(C)C)NC(=O)c1cc(OC)cc(OC)c1</smiles>

S5<smiles>CC(I)C(=O)NC(C)c1cccc2ccccc12</smiles>

S2<smiles>[CH2]C(=O)NC(C)c1cccc2cc(C)c(C)cc12</smiles>
S6<smiles>CC(NC(=O)C=O)c1cccc2ccccc12</smiles>

S3<smiles>CC(=O)NC(C)c1cccc2cc(C)c(C)cc12</smiles>

S7<smiles>CCOC(=O)C(NC(=O)c1cc([N+](=O)[O-])cc([N+](=O)[O-])c1)c1ccccc1</smiles>

S4<smiles>CC(=O)NC(C)c1cccc2cc(C)c(C)cc12</smiles>

S8<smiles>CC(=O)OC(=O)NC(C(=O)Nc1ccccc1)c1ccccc1</smiles>

s9<smiles>CC(=O)NC(C(=O)Nc1ccccc1)c1ccccc1</smiles>

S10<smiles>O=C(NC(CO)c1ccccc1)c1cc([N+](=O)[O-])cc([N+](=O)[O-])c1</smiles>

Figure 1. Structures of racemic samples. 
$\delta 0.5(\mathrm{t}, 2 \mathrm{H}), 1.2(\mathrm{~m}, 9 \mathrm{H}), 1.5(\mathrm{~m}, 2 \mathrm{H}), 2.9(\mathrm{q}, 2 \mathrm{H}), 3.9(\mathrm{q}$, $6 \mathrm{H}), 7.0-7.7(\mathrm{~m}, 12 \mathrm{H}), 8.41-8.43(\mathrm{~d}, 1 \mathrm{H}), 8.6(\mathrm{~d}, 2 \mathrm{H}), 9.1(\mathrm{t}$, 1H). IR (KBr) cm$~^{-1}: 3551,3413,3130,1742,1638,1618$, 1401.

Preparation of CSP 1 and Column Packing: A flask equipped with a Dean-Stark trap and a condenser was charged with Spherical silica gel $(2.72 \mathrm{~g}$, particle size: $5 \mu \mathrm{m})$ and toluene $(40 \mathrm{~mL})$. After heating the heterogeneous mixture under reflux until azeotropic removal of water was complete, compound 2 ( $0.60 \mathrm{~g}, 1.79 \mathrm{mmol})$, dissolved in 10 $\mathrm{mL}$ toluene, was added and then the whole mixture heated to reflux for $72 \mathrm{~h}$ under nitrogen. The silica gel was filtered and washed extensively with toluene, ethyl acetate, methanol, acetone, diethyl ether and hexane successively. Then the modified silica gel was slurried in methanol and packed into a $4.6 \mathrm{~mm}$ ID, $250 \mathrm{~mm}$ length stainless steel HPLC column using a conventional method ${ }^{8,9}$ with an Alltech HPLC Slurry Packer.

\section{Preparation of CSP 2.}

Preparation of [1,1'-binaphthalene]-2,2'-diyl bis((3(triethoxysilyl)propyl)carbamate) (3): $(R)-1,1^{\prime}-\mathrm{Bi}(2$-naphthol) (1.00 g, $3.46 \mathrm{mmol})$ and triethylamine $(0.97 \mathrm{~mL}, 6.92$ mmol) were dissolved in $100 \mathrm{~mL}$ of dry methylene chloride in a $250 \mathrm{~mL}$ two-necked round bottom flask. To the stirred solution, 3-(triethoxysilyl)-propyl isocyanate (1.81 mL, 6.92 mmol) were added. The whole reaction mixture was stirred for $12 \mathrm{~h}$ at room temperature, and then the solvent in the flask was evaporated. The residue was purified by column chromatography over silica gel with EtOAc/ $n$-hexane $/ \mathrm{CH}_{2} \mathrm{Cl}_{2}$ (1:4:10) to afford $3(1.50 \mathrm{~g}$, yield $55.5 \%)$ as a colorless high viscosity liquid. ${ }^{1} \mathrm{H}-\mathrm{NMR}\left(\mathrm{CDCl}_{3}\right) \delta 0.5(\mathrm{t}, 4 \mathrm{H}), 1.2(\mathrm{~m}$, $18 \mathrm{H}), 1.7(\mathrm{~m}, 4 \mathrm{H}), 2.9(\mathrm{~m}, 4 \mathrm{H}), 3.8(\mathrm{q}, 12 \mathrm{H}), 7.2-8.0(\mathrm{~m}$, $12 \mathrm{H})$. IR (KBr) cm $\mathrm{cm}^{-1}: 3552,3412,3130,1749,1637,1533$, 1401, $1222,1104$.
Preparation of CSP 2 and Column Packing. A flask equipped with a Dean-Stark trap and a condenser was charged with Spherical silica gel (3.20 g, particle size: $5 \mu \mathrm{m})$ and toluene $(50 \mathrm{~mL})$. After heating the heterogeneous mixture under reflux until the azeotropic removal of water was complete, compound 3 ( $0.80 \mathrm{~g}, 1.79 \mathrm{mmol})$, dissolved in 10 $\mathrm{mL}$ toluene, was added and then the whole mixture was heated to reflux for $72 \mathrm{~h}$ under nitrogen. The silica gel was filtered and washed extensively with toluene, ethyl acetate, methanol, acetone, diethyl ether, and hexane successively. Then the modified silica gel was slurried in methanol and packed into a $4.6 \mathrm{~mm}$ ID, $250 \mathrm{~mm}$ length stainless steel HPLC column using a conventional method with an Alltech HPLC Slurry Packer.

\section{Results and Discussion}

The preparation of CSP 1 is summarized in Scheme 1. The synthetic procedures outlined in Scheme 1 involved only three major steps: Esterification, silylation and bonding to silica gel. These simple reactions have been reported in literature previously., ${ }^{4,8-12}$ First, the mono-substituted compound 1 was a major product at low temperatures, but a disubstituted product also appeared. Because the yield of the esterification was bigger than the amidation (the first step of synthesis of CSP 3), the di- substituted one could be cleanly and easily removed by classical extraction with high $\mathrm{pH}$ $(\sim 13)$ of the aqueous solution. The preparation of CSP 2 is summarized in Scheme 2. The synthetic procedures outlined in Scheme 2 involved only two steps: Silylation and bonding to silica gel. The CSP 2 has a binaphthol group but no additional $\pi$-acidic group. CSP 3 and CSP 4 were easily prepared by following the previously reported method ${ }^{4}$ under the similar synthetic conditions with different starting

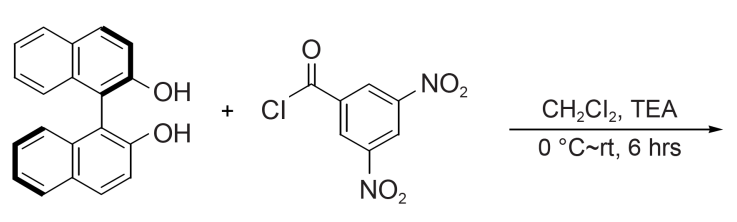

R-1, 1'-Bi(2-naphthol) 3,5-dinitrobenzoyl chloride
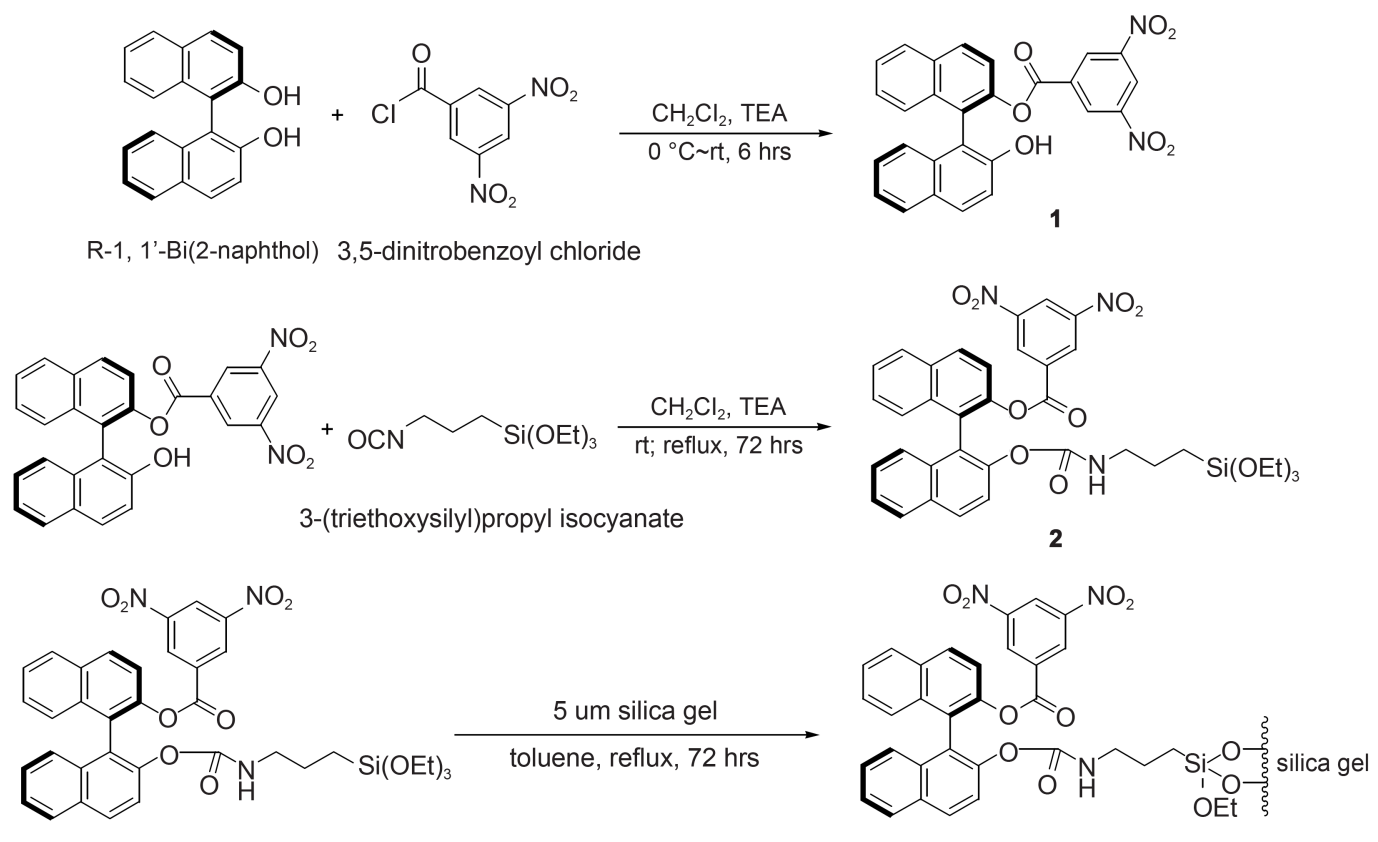

$\operatorname{CSP} 1$

Scheme 1 


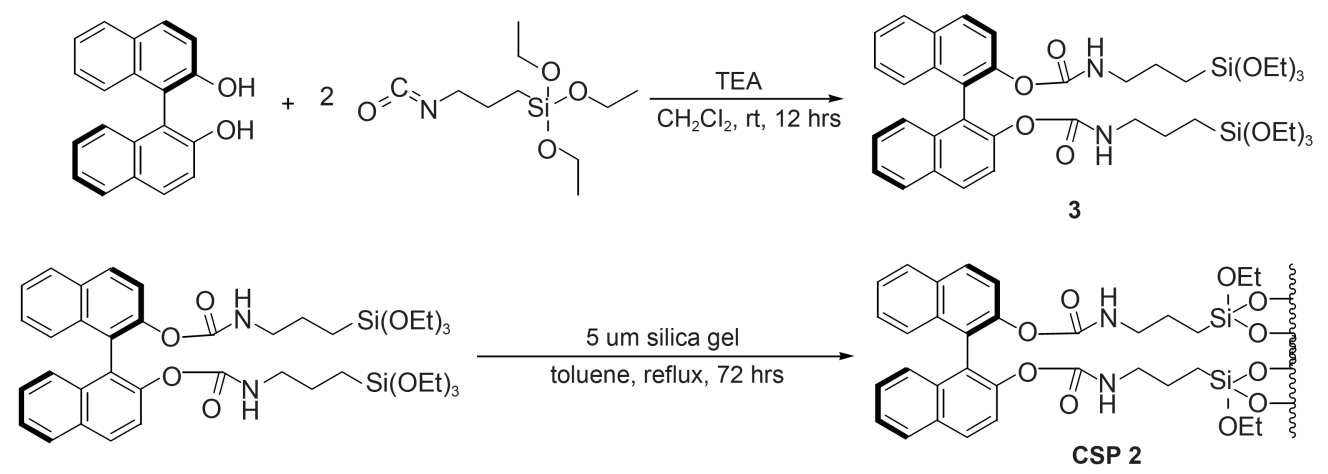

Scheme 2

Table 1. Elemental analysis of CSP 1, CSP 2, CSP 3, and CSP 4

\begin{tabular}{lcccc}
\hline & $\mathrm{C}$ & $\mathrm{N}$ & based on $\mathrm{N}$ & based on C \\
\hline CSP 1 & $7.718 \%$ & $1.043 \%$ & $0.248 \mathrm{mmol} / \mathrm{g}$ & $0.207 \mathrm{mmol} / \mathrm{g}$ \\
CSP 2 & $6.078 \%$ & $0.599 \%$ & $0.214 \mathrm{mmol} / \mathrm{g}$ & $0.181 \mathrm{mmol} / \mathrm{g}$ \\
CSP 3 & $6.238 \%$ & $1.155 \%$ & $0.165 \mathrm{mmol} / \mathrm{g}$ & $0.167 \mathrm{mmol} / \mathrm{g}$ \\
CSP 4 & $7.233 \%$ & $1.141 \%$ & $0.204 \mathrm{mmol} / \mathrm{g}$ & $0.215 \mathrm{mmol} / \mathrm{g}$ \\
\hline
\end{tabular}

material of CSP 1 or CSP 2 . The covalently modified silica gels were packed into $150 \mathrm{~mm}$ length $\times 4.6 \mathrm{~mm}$ I.D. $(2.1$ $\mathrm{mm}$ for CSP 1) stainless steel columns using conventional methods. $^{10,11}$

All CSPs possess two $\pi$-basic naphthyl moieties; furthermore, CSP 1 and CSP 3 have an additional $\pi$-acidic 3,5dinitrobenzoyl group. The results of elemental analysis of CSP 1-CSP 4 are shown in Table 1. The amount of covalent bonding of each selector to silica was about $0.2 \mathrm{mmol}$ per one gram of silica gel. This is similar result of previous work. ${ }^{1,9}$

Some examples of the enantiomeric separation of 2,2,2trifluoro-1-(9-anthryl)ethanol (S1) on CSP 1 and CSP 3, and S11 on CSP 2 and CSP 4 is shown in Figure 2.

As shown in Figure 2, both CSP 1 and CSP 3 showed good resolution, and CSP 1 showed better resolution than CSP 3. Racemic phenylglycinol- $N$-3,5-dinitrobenzamide (S11) was slightly separated on CSP 2 and CSP 4. The chromatographic separation data of the 11 racemic samples on CSP 1-CSP 4 are summarized in Table 2.

As shown in Table 2, nine racemic compounds were separated on CSP 1 and all chiral samples used in this study were separated on CSP 3, while only four chiral compounds were separated on CSP 2 and CSP 4. The dinitrobenzoyl (DNB) group containing CSPs (CSP 1 and CSP 3) showed much broader and higher selectivity than the previously reported binaphthol, binaphthalene, or diphenylethanediamine derived CSPs. ${ }^{9,12,13}$ In the resolution of various 23 chiral samples on the first reported four binaphthol derived CSPs (CSP 5-8), CSP 5 resolved 11 racemates from 23 samples with a maximum selectivity factor $(\alpha)$ of $1.12,9 / 23$ (maximum a value is 1.062) on CSP 6, 13/23 (maximum $\alpha$ value is 1.11) on CSP 7, and 14/23 (maximum $\alpha$ value is 1.18) on CSP 8. ${ }^{9}$ In the case of newly developed a 1,1'-bi-2-

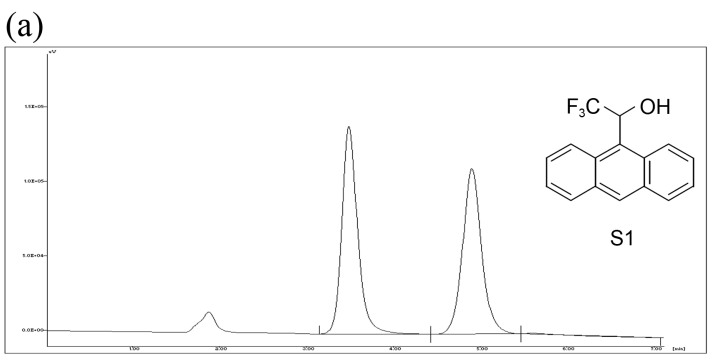

(b)

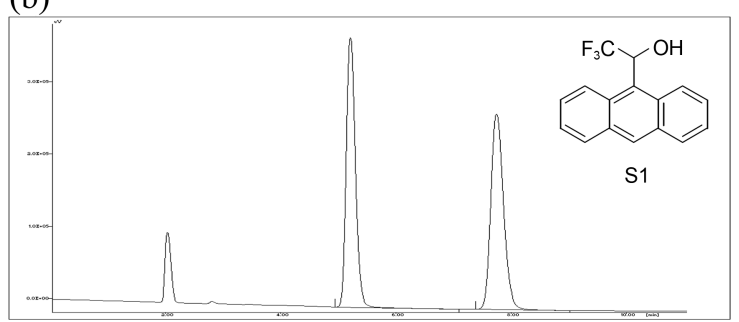

(c)

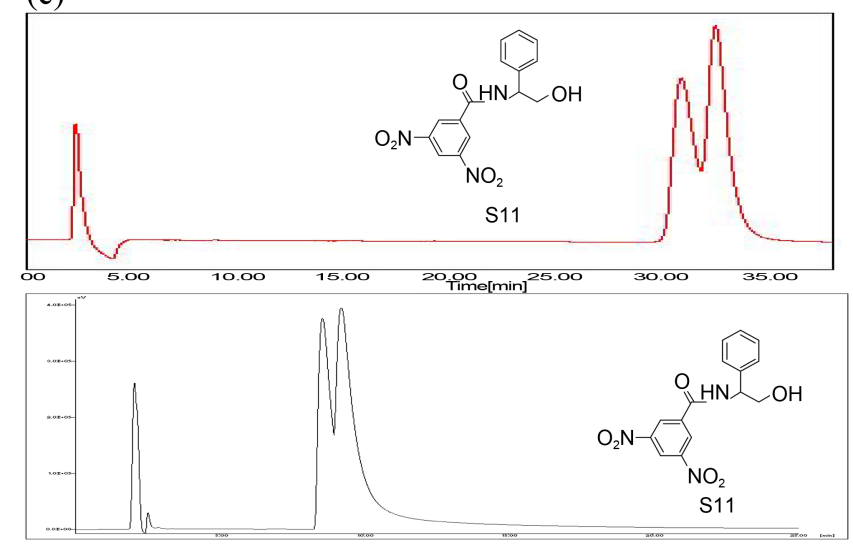

Figure 2. (a) Resolution of S1 on CSP 1. Column size; $2.1 \mathrm{~mm} \times$ $15 \mathrm{~cm}$, Eluent; $10 \%$ IPA in hexane, Flow rate; $0.3 \mathrm{~mL} / \mathrm{min}$, Detection at $254 \mathrm{~nm}$. (b) Resolution of S1 on CSP 3. Column size; $4.6 \mathrm{~mm} \times 15 \mathrm{~cm}$, Eluent; $10 \%$ IPA in hexane, Flow rate; $1 \mathrm{~mL} / \mathrm{min}$, Detection at $254 \mathrm{~nm}$. (c) Resolution of S11 on CSP 2 and CSP 4. Column size; $4.6 \mathrm{~mm} \times 15 \mathrm{~cm}$, Eluent; 10\% IPA in hexane, Flow rate; $1 \mathrm{~mL} / \mathrm{min}$, Detection at $254 \mathrm{~nm}$.

naphthol derived CSP 1 by connecting with silica gel at a hydroxy $(\mathrm{OH})$ group of binaphthol showed much better resolution than the previous four binaphthol derived CSPs. 
Table 2. Separation data for eleven racemic compounds on CSP 1-4 as a function of different mobile phase compositions ${ }^{a}$

\begin{tabular}{|c|c|c|c|c|c|c|c|c|c|c|c|c|c|c|c|c|}
\hline \multirow{4}{*}{ Sample } & \multicolumn{4}{|c|}{ CSP 1} & \multicolumn{4}{|c|}{ CSP 2} & \multicolumn{4}{|c|}{ CSP 3} & \multicolumn{4}{|c|}{ CSP 4} \\
\hline & \multicolumn{16}{|c|}{ Eluent (percent of IPA in Hexane) } \\
\hline & \multicolumn{2}{|c|}{2.5} & \multicolumn{2}{|c|}{10} & \multicolumn{2}{|c|}{2.5} & \multicolumn{2}{|c|}{10} & \multicolumn{2}{|c|}{2.5} & \multicolumn{2}{|c|}{10} & \multicolumn{2}{|c|}{2.5} & \multicolumn{2}{|c|}{10} \\
\hline & $k$ & $\alpha$ & $k$ & $\alpha$ & $k$ & $\alpha$ & $k$ & $\alpha$ & $k$ & $\alpha$ & $k$ & $\alpha$ & $k$ & $\alpha$ & $k$ & $\alpha$ \\
\hline S1 & 2.87 & 2.16 & 0.93 & 1.84 & 2.66 & 1.05 & 0.82 & 1.00 & 4.74 & 1.68 & 1.88 & 1.75 & 2.83 & 1.00 & 0.79 & 1.00 \\
\hline S2 & 5.16 & 1.04 & 1.28 & 1.00 & 5.53 & 1.00 & 1.05 & 1.00 & 13.96 & 1.48 & 4.67 & 1.65 & 5.51 & 1.00 & 1.40 & 1.00 \\
\hline S3 & 3.75 & 1.05 & 0.96 & 1.00 & 4.01 & 1.00 & 0.75 & 1.00 & 10.37 & 1.63 & 3.48 & 1.86 & 3.93 & 1.00 & 0.98 & 1.00 \\
\hline S4 & 5.79 & 1.00 & 1.83 & 1.00 & 5.30 & 1.00 & 1.23 & 1.00 & 11.69 & 1.08 & 4.2 & 1.05 & 6.94 & 1.00 & 1.86 & 1.00 \\
\hline S5 & 2.16 & 1.00 & 2.28 & 1.09 & 2.80 & 1.00 & 0.61 & 1.00 & 2.87 & 1.06 & 2.28 & 1.09 & 2.61 & 1.00 & 0.72 & 1.00 \\
\hline S6 & 3.49 & 1.07 & 1.13 & 1.09 & 2.43 & 1.00 & 0.10 & 1.00 & 13.49 & 1.17 & 4.42 & 1.20 & 2.92 & 1.00 & 0.76 & 1.00 \\
\hline S7 & 4.05 & 1.10 & 1.22 & 1.08 & 3.62 & 1.00 & 1.00 & 1.00 & 14.43 & 1.12 & 4.89 & 1.15 & 3.52 & 1.00 & 0.94 & 1.00 \\
\hline S8 & 7.86 & 1.08 & 6.17 & 1.07 & 5.57 & 1.00 & 1.58 & 1.00 & 23.78 & 1.02 & 7.26 & 1.05 & 6.14 & 1.06 & 1.53 & 1.00 \\
\hline S9 & 2.35 & 1.10 & 0.88 & 1.00 & 2.20 & 1.12 & 0.54 & 1.00 & 6.89 & 1.19 & 1.45 & 1.12 & 2.96 & 2.08 & 0.66 & 1.71 \\
\hline S10 & 3.77 & 1.07 & 1.32 & 1.00 & 2.22 & 1.11 & 0.53 & 1.00 & 8.41 & 1.25 & 1.77 & 1.19 & 3.16 & 2.63 & 0.66 & 2.13 \\
\hline S11 & 12.9 & 1.00 & 1.65 & 1.00 & 13.6 & 1.07 & 1.41 & 1.00 & 3.57 & 1.13 & 3.62 & 1.14 & 22.2 & 1.07 & 3.65 & 1.10 \\
\hline
\end{tabular}

${ }^{a}$ Flow rate for CSP 1 = $0.3 \mathrm{~mL} / \mathrm{min} . ;$ $254 \mathrm{~nm}$.

(9/11, maximum a value is 1.84) The reason for these broader and higher selectivities on the dinitrobenzoyl (DNB) group containing CSPs is related to the additional face-toface $\pi-\pi$ interaction between the $\pi$-acidic DNB group on the CSP and the $\pi$-basic group on the chiral samples. ${ }^{4,11}$

In comparison of the two 3,5-DNB-derived CSPs, CSP 3 showed better resolution than CSP 1. The reason for these results cannot be explained clearly at this moment, but it can be assumed that the amide hydrogen in the CSP 3 plays an important role (for example, additional hydrogen bonding with chiral samples) under these resolutions. There are some interesting points in this resolution. Samples 9 and 10 (S9, S10) were separated on all four CSPs used in this study, but showed much better resolution on CSP 4.

In conclusion, two new 1,1'-bi-2-naphthol-derived CSPs (CSP 1 and CSP 2) were very easily prepared by following a simple two- or three-step synthetic procedure. These binaphthol-derived CSPs were prepared much easier than previous binaphthol CSPs and demonstrated improved selectivity over the previously reported CSP. In general, the ease and simplicity of the preparation process of the CSP is important from a practical standpoint. For an exact comparison of the two CSPs and the previously reported two 1,1'-binaphthyl-2,2'-diamine derived CSPs (CSP 3 and CSP 4), the CSP 3 and CSP 4 were prepared again; the chiral separation result of 11 chiral compounds were then compared on these four columns. The two dinitrobenzoyl (DNB) groups containing CSPs (CSP 1 and CSP 3) showed much better results than CSP 2 and CSP 4. The CSP 3 showed better results than CSP 1 in the chiral separation of the tested samples.

Acknowledgments. This research was supported by Kyungpook National University Research Fund, 2008.

\section{References}

1. Gasparrini, F.; Misiti, D.; Villani, C. J. Chromatogr. A 2001, 906, 35-50.

2. Gopalaiah, K.; Kagan, H. B. Chem. Rev. 2011, 111, ASAP.

3. Pirkle, W. H.; Pochapsky, T. C. Chem. Rev. 1989, 89, 347-362.

4. Ryoo, J. J.; Armstrong, D. W. Bull. Korean Chem. Soc. 2007, 28 , 2103-2106.

5. Zhang, D.; Li, F.; Hyun, M. H. Pharmazie 2007, 62, 258-261.

6. Lu, Z.; Zhang, Y.; Wulff, W. D. J. Am. Chem. Soc. 2007, 129, 7185-7194.

7. Noyori, R.; Tomino, I.; Yamada, M.; Nishizawa, M. J. Am. Chem. Soc. 1984, 106, 6717-6725.

8. Bao, J.; Wulff, W. D.; Dominy, J. B.; Fumo, M. J.; Grant, E. B.; Rob, A. C.; Whitcomb, M. C.; Yeung, S. M.; Ostrander, R. L.; Rheingold, A. L. J. Am. Chem. Soc. 1996, 118, 3392-3405.

9. Yoshihisa, S.; Tomohiko, Y.; Toshio, S. J. Chromatogr. A 1998, $813,35-45$.

10. Guiochon, G.; Farkas, T.; Guan-Sajonz, H.; Koh, J. H.; Sarker, M.; Stanley, B. J.; Yun, T. J. Chromatogr. A 1997, 762, 83-88.

11. Ryoo, J. J.; Kim, T. H.; Im, S. H.; Jeong, Y. H.; Park, J. Y.; Choi, S. H.; Lee, K. P.; Park, J. H. J. Chromatogr. A 2003, 987, 429-438.

12. Uray, G.; Maier, N. M.; Niederreiter, K. S.; Spitaler, M. M. J. Chromatogr. A 1998, 799, 67-81.

13. Lindner, W.; Uray, G.; Steiner, U. J. Chromatogr. A 1991, 553, 373-381. 